\title{
Role of C5 in the development of airway inflammation, airway hyperresponsiveness, and ongoing airway response
}

\author{
Tao Peng, ${ }^{1}$ Liming Hao, ${ }^{2}$ Joseph A. Madri, ${ }^{2}$ Xiao Su, ${ }^{1}$ Jack A. Elias, ${ }^{3}$ \\ Gregory L. Stahl, ${ }^{4}$ Stephen Squinto, ${ }^{1}$ and Yi Wang ${ }^{1}$ \\ ${ }^{1}$ Alexion Pharmaceuticals Inc., Cheshire, Connecticut, USA. ${ }^{2}$ Department of Pathology and ${ }^{3}$ Department of Internal Medicine, \\ Yale University School of Medicine, New Haven, Connecticut, USA. ${ }^{4}$ Department of Anesthesiology, \\ Perioperative and Pain Medicine, Brigham and Women's Hospital, Boston, Massachusetts, USA.
}

\begin{abstract}
The role of complement component $\mathrm{C} 5$ in asthma remains controversial. Here we examined the contribution of $\mathrm{C} 5$ at 3 critical checkpoints during the course of disease. Using an $\mathrm{mAb}$ specific for $\mathrm{C} 5$, we were able to evaluate the contribution of $\mathrm{C} 5$ during (a) the initiation of airway inflammation, (b) the maintenance of airway hyperresponsiveness (AHR), and (c) sustainment of an ongoing airway response to allergen provocation. Our results indicate that $\mathrm{C} 5$ is probably activated intrapulmonarily after infections or exposures to allergen and C5 inhibition has profound effects at all 3 critical checkpoints. In contrast to an earlier report, C5-deficient mice with established airway inflammation did not have elevated AHR to nonspecific stimuli. In the presence of airway inflammation, C5a serves as a direct link between the innate immune system and the development of AHR by engaging directly with its receptors expressed in airways. Through their powerful chemotactic and cell activation properties, both $\mathrm{C} 5 \mathrm{a}$ and $\mathrm{C} 5 \mathrm{~b}-9$ regulate the downstream inflammatory cascade, which results in a massive migration of inflammatory cells into the bronchial airway lumen and triggers the release of multiple harmful inflammatory mediators. This study suggests that targeting $\mathrm{C5}$ is a potential clinical approach for treating patients with asthma.
\end{abstract}

\section{Introduction}

Asthma is characterized by a combination of chronic airway inflammation, airway obstruction, and airway hyperresponsiveness (AHR) to various stimuli. It is thought to be mediated primarily by adaptive immune responses mediated by allergen-specific $\mathrm{CD}^{+} \mathrm{T}$ cells, Th2 cytokines, and allergen specific IgE, which lead to pulmonary inflammation and AHR.

Complement and its activated components, which form a central core of innate immune defense against bacterial, viral, and fungal invasions (1-3), can be activated through the classical pathway, the alternative pathway, and the lectin pathway (4). All 3 activation pathways converge at complement component $\mathrm{C} 5$ prior to the generation of $\mathrm{C} 5 \mathrm{a}$ and $\mathrm{C} 5 \mathrm{~b}-9$, both of which induce potent biological responses, including tissue injury, inflammation, anaphylatoxic responses, and cell lysis at very low concentrations (5). In addition, $\mathrm{C} 5$ can be activated after allergen exposure (6). Recent data from animal models of allergic asthma suggest that activated complement components, such as C5a, provide a critical link between innate and adaptive immunity (7). However, controversies remain regarding the involvement of $\mathrm{C} 5$ and its activated components in the pathogenesis of asthma (7-10).

\footnotetext{
Nonstandard abbreviations used: AHR, airway hyperresponsiveness; BALF, bronchoalveolar lavage fluid; C5aR, C5a receptor; C5d, C5-deficient; Cdyn, dynamic lung compliance; C5s, C5-sufficient; DCP, double-chamber plethysmograph; EAR, earlyphase airway response; IC, immune complex; LAR, late-phase airway response; Mch, methacholine; rmC5a, recombinant mouse C5a; RL, lung resistance; sRaw, specific airway resistance; $\operatorname{sIgG}$, serum IgG.

Conflict of interest: Yi Wang, Tao Peng, Xiao Su, and Stephen Squinto are fully employed by Alexion Pharmaceuticals Inc., which developed anti-human C5 mAbs. Citation for this article: J. Clin. Invest. 115:1590-1600 (2005). doi:10.1172/JCI22906.
}

Several experimental models for bronchial asthma have indicated that $\mathrm{C} 5$ and its activated components are involved in the development of airway inflammation and bronchoconstriction. Studies with various complement inhibitors markedly reduced AHR or airway inflammation in rodents $(9,10)$. The potential involvement of C5 activation was also extended to clinical observations that the severity of clinical symptoms was correlated with the extent of C5 activation (11). On the other hand, studies have shown that C5 deficiency leads to increased susceptibility to allergeninduced AHR in mice, and this finding is supported by evidence of decreased production of IL-12, a key Th1 cytokine reported to modulate the pathogenesis of asthma (7). The key question at the center of the debate is whether C5 and its activated components are proinflammatory or antiinflammatory during the sensitization phase and the effector phase of the pathogenesis as suggested, respectively, by studies of C5-deficient (C5d) animals (7) and experiments involving intervention during the course of disease $(9,10)$. Another key issue is whether intrapulmonarily activated complement components play a significant role in the pathogenesis and overcome the potential antiinflammatory effect of activated C5 components on the adaptive immune system (7).

This study examines the contribution of $\mathrm{C} 5$ and its activated components at 3 critical points during the course of disease and outlines the key mechanisms of this essential component of the innate immune system in the pathogenesis of asthma. We have performed comprehensive analyses of lower airway function and quantified multiple parameters of airway inflammation. We first examined whether C5 contributes to the initiation of airway inflammation, 1 of the 2 hallmarks of asthma's pathogenesis. Second, we sought to determine the contribution of $\mathrm{C} 5$ in maintaining AHR to nonspecific stimuli when substantial airway damage and significant 
A

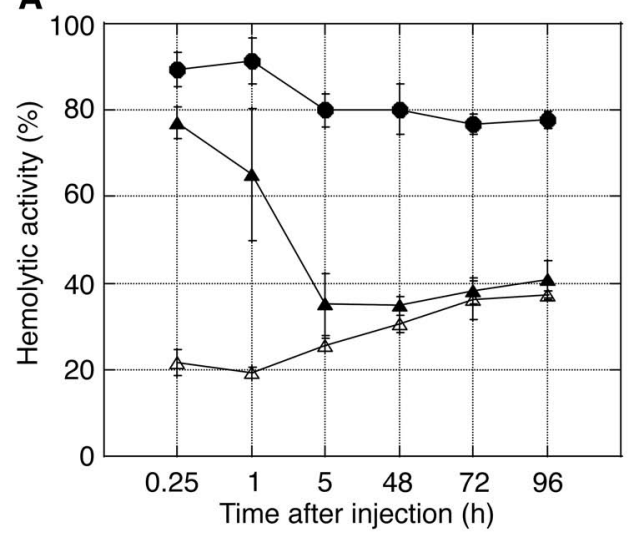

B

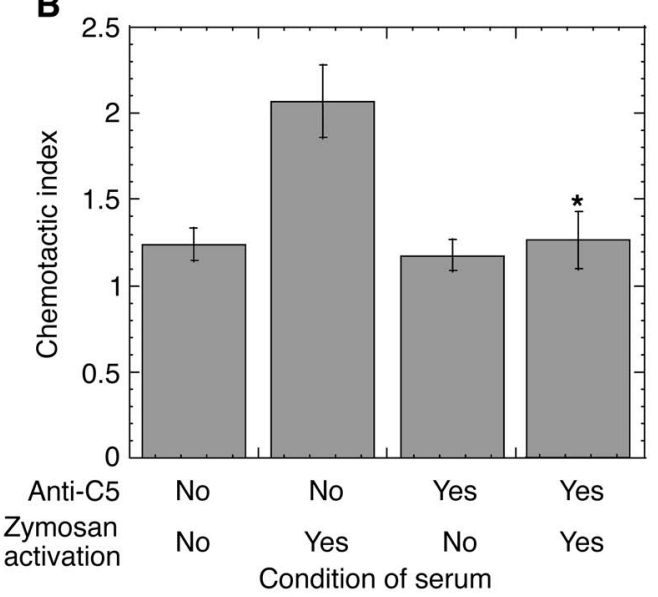

Figure 1

Blocking the generation of $\mathrm{C} 5 \mathrm{a}$ and $\mathrm{C} 5 \mathrm{~b}-9$ by anti-C5 mAb, BB5.1. (A) Pharmacodynamic profile of inhibition of C5b-9-mediated hemolysis from animals given a single i.v. or i.p. injection of anti-C5 mAb (40 mg/kg) or control $\mathrm{mAb}(40 \mathrm{mg} / \mathrm{kg})$. Filled circles represent data from 4 control animals that were given control $\mathrm{mAb}$ either i.v. $(n=2)$ or i.p. $(n=2)$. Filled triangles represent i.p. anti-C5 mAb-treated animals $(n=3)$. Open triangles represent i.v. anti-C5 mAb-treated animals $(n=3)$. (B) C5a-mediated neutrophil migration was analyzed in an in vitro assay. Zymosan-activated sera (20\%) was used as the source of C5a. Anti-C5 mAb $(100 \mu \mathrm{g} / \mathrm{ml})$ was added to serum sample prior to zymosan activation as indicated $(n=8)$. ${ }^{*} P<0.05 \mathrm{com}$ pared with zymosan-activated serum samples. BALB/c mice were used in the experiments reported in all figures except Figure 4, C and D.

airway inflammation are already present in subjects who had previously experienced a severe airway response. We further evaluated the role of $\mathrm{C} 5$ in the development of AHR in C5d mice in order to compare the results directly with those in a previous report (7). Third, we examined the involvement of $\mathrm{C} 5$ in sustaining an ongoing airway response to allergen provocation. Finally, we evaluated the contribution of intrapulmonary activation of $\mathrm{C} 5$, in particular the role of C5a versus C5b-9 in the pathogenesis.

\section{Results}

Blocking the generation of $\mathrm{C} 5 \mathrm{a}$ and $\mathrm{C} 5 \mathrm{~b}-9$ by anti-C5 $\mathrm{mAb}, \mathrm{BB} 5.1$. Functional inhibition of complement component $\mathrm{C} 5$ by anti-C5 $\mathrm{mAb}$ was determined by the pharmacodynamic profile of blocking serum C5b-9-mediated hemolytic activity (Figure 1A). In addition, C5a-mediated neutrophil migration was determined in an in vitro assay (Figure 1B).

The pharmacodynamics of C5 inhibition by a single i.v. injection of anti-C5 mAb, BB5.1, is very different from the pharmacodynamics of a single i.p. injection during the first 5 hours after administration. More than $80 \%$ of C5b-9-mediated hemolysis was inhibited within 1 hour of i.v. administration of BB5.1, compared with less than $30 \%$ of inhibition by i.p. injection. Administration of anti-C5 $\mathrm{mAb}$ continued blocking more than $60 \%$ of hemolysis between 5 and 48 hours after i.v. injection, while approximately $56 \%$ of hemolysis was blocked during this period after i.p. injection. Serum samples harvested from control mAb-treated mice had more than $80 \%$ of normal hemolytic activity during the entire period of the study (Figure $1 \mathrm{~A}$ ). Hemolytic activity gradually returned to the normal range 1 week after a single injection of anti-C5 mAb (data not shown).

We next used a well-described in vitro neutrophil migration assay (12) to test the ability of anti-C5 mAb to block the generation of $\mathrm{C5a}$ (Figure 1B). Inhibition of serum samples with anti-C5 mAb prior to zymosan activation significantly blocked human neutrophil chemotaxis, which was mediated by the presence of C5a after zymosan activation, as seen in zymosan-activated control sera (Figure 1B).

Since BB5.1 blocked the generation of both $\mathrm{C} 5 \mathrm{a}$ and C5b-9 and did not bind to C5a directly (data not shown), this anti-C5 $\mathrm{mAb}$ is probably specific to C5 convertase.

Development of severe airway response in OVA-sensitized animals and definition of 3 critical points. OVA-sensitized animals immediately developed a severe airway response after aerosol challenge with 5\% OVA (Figure 2A). A typical airway response to aerosol provocation with allergen consisted of an early-phase airway response (EAR), which is mediated by the release of histamine by mast cells (13), and a late-phase airway response (LAR), which is mediated by the combination of effects of infiltration of inflammatory cells, edema, and bronchial constriction (13). The airway response after 5\% OVA provocation was monitored by a noninvasive double-chamber plethysmograph (DCP) in each mouse to measure the longitudinal changes in specific airway resistance (sRaw), which indicates the appearance of EAR and LAR (Figure 2A). EAR typically constituted a brief elevation of sRaw 15 minutes after 5\% OVA provocation, and LAR was typically observed 5 hours later, consistent with an earlier report (14). Both EAR and LAR have magnitudes between 5 - and 30 -fold relative to baseline. The severe airway response during the peak of LAR was generally evident, as animals showed obvious labored breathing.

Since inhibition of $\mathrm{C} 5$ can be achieved by the administration of anti-C5 $\mathrm{mAb}$, we sought to dissect the involvement of $\mathrm{C} 5$ and its activated components at 3 critical points (Figure 2B) during the disease. The 3 critical checkpoints were: initiation of airway inflammation, 1 of the 2 hallmarks of asthma's pathogenesis (checkpoint 1 ); development of AHR to nonspecific stimuli, another hallmark of asthma (checkpoint 2); and sustainment of an ongoing airway response after allergen provocation (checkpoint 3 ). Both checkpoint 2 and checkpoint 3 were designed to evaluate the contribution of $\mathrm{C} 5$ in subjects that had experienced severe airway response previously (Figure 2B) and had established airway inflammation. We conducted checkpoint 3 studies during an ongoing airway response after allergen provocation by administering treatment via either an i.v. or aerosol route during the EAR. Functions of lower airways were evaluated and airway inflammation quantified during the peak of LAR, 5 hours after $5 \%$ OVA provocation.

Checkpoint 1: involvement of $\mathrm{C} 5$ in the initiation of airway inflammation. C5 inhibition at checkpoint 1, at the time of repeat aerosol sensitization with $1 \%$ OVA, had no significant impact on serum levels of OVA-specific IgE or IgG on day 32 (Figure 3A). Corticosteroid-treated mice also had elevated levels of OVA-specific IgE and 


\section{A}

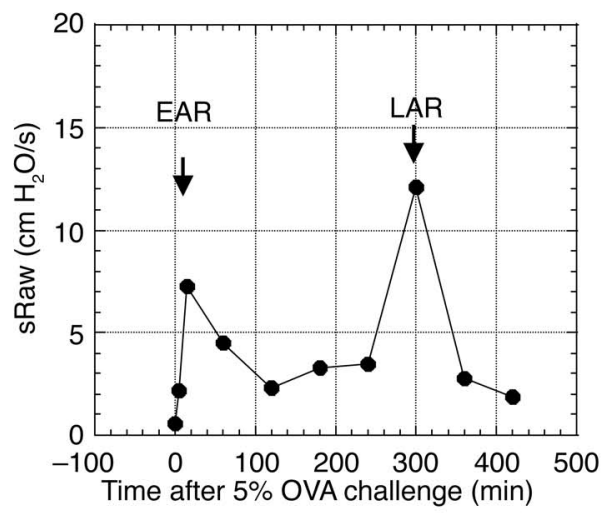

B

Induction of disease (days after initial immunization on day 0 )

$\left.\begin{array}{lll}\text { Day 28-30: Initiation of } \\ \text { airway inflammation } \\ \text { with daily aerosol } \\ \text { sensitization with } \\ 1 \% \text { OVA }\end{array} \rightarrow \begin{array}{l}\text { Day 32: Induction of } \\ \text { first severe airway } \\ \text { response with aerosol } \\ \text { provocation with } \\ 5 \% \text { OVA }\end{array}\right) \begin{aligned} & \text { Day 33: Untreated mice } \\ & \text { recover from first airway } \\ & \text { response on day } 32 . \\ & \text { Animals have established } \\ & \text { airway inflammation. }\end{aligned}$

\begin{tabular}{|c|c|}
\hline $\begin{array}{l}\text { Checkpoint } 1 \text { : } \\
\text { Initiation of airway } \\
\text { inflammation (C5 } \\
\text { inhibition on days } \\
25,29 \text {, and } 31 \text { ) }\end{array}$ & $\begin{array}{l}\text { Evaluation of the } \\
\text { development of first } \\
\text { airway response to } \\
\text { allergen provocation } \\
\text { and the degree of } \\
\text { airway inflammation }\end{array}$ \\
\hline
\end{tabular}

\begin{tabular}{|ll|}
\hline Checkpoint 2: & Evaluation of \\
Development of & AHR to aerosol \\
AHR in mice with & Mch challenges \\
established airway & $\rightarrow$ \\
inflammation (C5 & on day 35 \\
inhibition on day 33) & \\
\hline
\end{tabular}
response. Untreated mice were aerosol challenged again with $5 \%$ OVA.

\begin{tabular}{|ll|}
\hline Checkpoint 3: & Evaluation of \\
During an ongoing & functions of lower \\
airway response & $\rightarrow$ \\
(C5 inhibition & airways during LAR \\
during EAR) & and analysis of BALF \\
\hline
\end{tabular}

\section{Figure 2}

Airway response to OVA provocation and definition of 3 critical checkpoints. (A) Representative DCP results indicating the longitudinal changes in sRaw. After aerosol challenge with 5\% OVA for 10 minutes, the appearance of EAR was observed at 15 minutes, followed by the LAR at 5 hours. (B) Three critical points during the course of disease. Checkpoint 1: Anti-C5 mAb was given by i.p. injection on days 25, 29, and 31 . Analysis of lower airway functions and quantification of airway inflammation were evaluated 5 hours after aerosol challenge with $5 \%$ OVA on day 32 . Checkpoint 2: Anti$\mathrm{C} 5 \mathrm{mAb}$ was given by i.p. injection on day 33, after recovery from the first airway response on day 32 . AHR was assessed by evaluating the changes in RL and Cdyn from baseline in response to aerosol Mch challenges on day 35. Checkpoint 3: C5 inhibition was given by either i.v. or aerosol administration of BB5.1 during EAR on day 35. Lower airway function was determined during the peak of LAR, 5 hours after $5 \%$ OVA provocation.

IgG that may have been generated long before the corticosteroid or anti-C5 mAb was administered on day 25. Sham-treated mice had a negligible level of OVA-specific IgG or IgE. As a result of repeated administration (i.p.) of anti-C5 mAb, approximately $80 \%$ of C5b-9mediated hemolysis was inhibited on day $32(21.1 \% \pm 4 \%)$, while the hemolytic activity seen in control $\mathrm{mAb}$ - and corticosteroidtreated mice was normal $(92.7 \% \pm 6.8 \%)$.

We examined two key functions of lower airways by tracheal cannulation at 5 hours after $5 \%$ OVA provocation. The increase in lung resistance (RL) is commonly associated with significant obstruction of lower airways (Figure 3B), and a decrease in dynamic lung compliance (Cdyn) is associated with the loss of lung elasticity, an important characteristic of the return to normal volume after the pressure changes seen in a normal lung (Figure 3C). The significantly increased RL and reduced Cdyn seen in control mAb-treated mice indicated an increased airway obstruction (Figure 3, B and C). Sham-treated mice showed normal ranges of RL and Cdyn at 5 hours after exposure to aerosol PBS solution.
Correlating with its potent antiinflammatory and antiasthmatic activities, corticosteroid treatment at checkpoint 1 significantly blocked the increase of RL and prevented the loss of Cdyn. Similarly, C5 inhibition at checkpoint 1 markedly attenuated the increase of RL 5 hours after allergen provocation with an RL of $1.66 \pm 0.2 \mathrm{~cm}$ $\mathrm{H}_{2} \mathrm{O} / \mathrm{ml} / \mathrm{s}$, compared with the $\mathrm{RL}$ of $2.17 \pm 0.33 \mathrm{~cm} \mathrm{H}_{2} \mathrm{O} / \mathrm{ml} / \mathrm{s}$ seen in control mAb-treated animals (Figure 3B). C5 inhibition also slightly reduced the loss of Cdyn (Figure 3C).

Double-blind histological analysis (Figure 3D) of lung tissue confirmed data from the function analysis and demonstrated that control $\mathrm{mAb}$-treated $\mathrm{BALB} / \mathrm{c}$ mice had severe perivascular and peribronchial infiltration of inflammatory cells (Figure $3 \mathrm{~F}$ ). A significant reduction in histology score (see Methods) was observed in corticosteroid-treated mice (Figure 3G), while a less impressive reduction was observed in anti-C5 mAb-treated mice (Figure $3 \mathrm{H}$ ). As is the case in severe allergic asthma in humans, eosinophils were the predominant inflammatory cells (more than 50\%) in the perivascular and peribronchial lesions along the airways (Figure 3, F-H). 

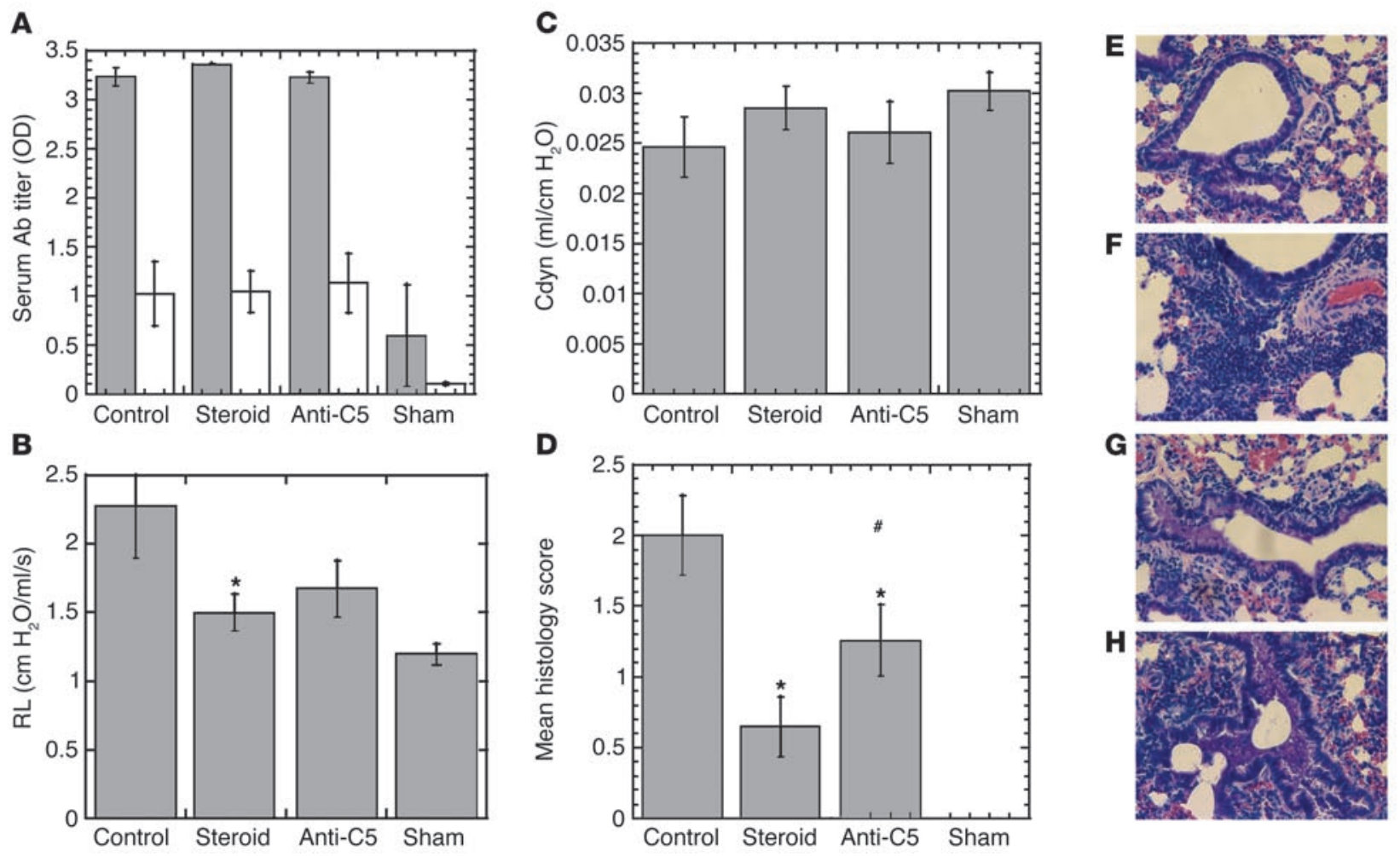

\section{Figure 3}

Contribution of C5 at checkpoint 1, the initiation of airway inflammation. (A) Mean serum OVA-specific antibody titers of 5-6 mice on day 32 were measured by ELISA. Gray bars: OVA-specific IgG; white bars: OVA-specific IgE. Also on day 32, animals were trachea cannulated for the measurement of $R L(B)$ and Cdyn (C) 5 hours after $5 \%$ aerosol OVA provocation, which was followed by histological analyses of lung (D). The mean histology score for control mAb-treated mice $(\mathbf{F})$ was $2 \pm 0.28$ compared with $0.64 \pm 0.21$ for corticosteroid-treated animals $(\mathbf{G})$ and $1.25 \pm 0.25$ for anti-C5 mAb-treated animals $(\mathbf{H})$ compared with the total lack of inflammation (score: 0$)$ seen in sham-treated mice $(\mathbf{E}) .{ }^{*} P<0.05$, corticosteroid- or anti-C5 mAb-treated compared with control mAb-treated mice; ${ }^{P} P<0.05$, corticosteroid-treated mice compared with animals treated with anti-C5 mAb. For clarity, all control mAb-treated animals are labeled as control in all following figures. Arrow indicates eosinophil infiltration. Giemsa stain, magnification $=\times 100$.

Checkpoint 2: contribution of C5 in the development of AHR in animals with established airway inflammation. In addition to airway inflammation, AHR to nonspecific stimuli is another hallmark of asthma. AHR was evaluated as changes in RL and Cdyn expressed as a percentage of baseline in response to increasing doses of aerosol methacholine (Mch) challenges (Figure 4, A and B).

Control $\mathrm{mAb}$-treated $\mathrm{BALB} / \mathrm{c}$ mice had significant increases in RL and significant losses of Cdyn during the course of aerosol Mch challenges, while sham-treated mice responded only modestly to aerosol Mch challenges. Two of 8 control mAb-treated mice died during challenge with high doses of aerosol Mch due to severe respiratory stress. In contrast, C5 inhibition prevented dramatic increases in RL and the reduction in Cdyn during the course of aerosol Mch challenges, similar to what occurred in animals treated with corticosteroid (Figure 4, A and B). The loss of Cdyn and increase in RL during the course of aerosol Mch challenges in anti-C5 mAb-and corticosteroid-treated mice compared with sham-treated mice were probably due to the presence of established airway inflammation at this checkpoint. There were no noticeable differences in the degree of airway inflammation among the 3 treated cohorts. Anti-C5 $\mathrm{mAb}$-treated mice had approximately $45 \%$ of normal hemolytic activity on day 35 .
In order to compare the present results of C5 inhibition at checkpoint 2 with data from a previous report (7), we examined whether native, intrinsic AHR is present in some strains of normal C5d mice, as previously reported $(15,16)$. Nonimmunized $\mathrm{C} 5 \mathrm{~d}$ AKR/J mice have dramatic airway responses to increasing doses of aerosol Mch challenges, with significant increases in RL relative to baseline $(84.2 \% \pm 16.7 \%$ with 20 puffs $\mathrm{Mch})$ and a significant reduction in Cdyn relative to baseline $(40 \% \pm 5.4 \%$ with 20 puffs of Mch), whereas C5d SWR/J and B10.D2-Hc ${ }^{0} 2^{d}-T 18^{c} /$ oSnJ (also known as B10D2.oSn) mice had minimal changes in $\mathrm{RL}$ and Cdyn, indistinguishable from levels in C5-sufficient (C5s) $\mathrm{BALB} / \mathrm{c}$ and $\mathrm{C} 57 \mathrm{BL} / 6$ mice, with maximal changes of only $15 \%$ in either RL or Cdyn from baseline $(n=3$ for each strain; data not shown). We next immunized and aerosol Mch challenged C5d B10D2.oSn mice, which do not have intrinsic AHR, in a manner identical to that for C5s BALB/c mice (Figure 4, A and B). Subgroups of sensitized C5d animals were also given either control or anti-C5 mAb treatment on day 33. C5d B10D2.oSn mice and C5s $\mathrm{BALB} / \mathrm{c}$ mice developed a similar degree of airway inflammation, with average histology scores of $1.91 \pm 0.37(n=6)$ and $2.25 \pm 0.25$ $(n=4)$, respectively. No noticeable histological difference was observed between anti-C5 mAb-and control mAb-treated C5d mice. In contrast to previously reported findings (7), increasing 
A

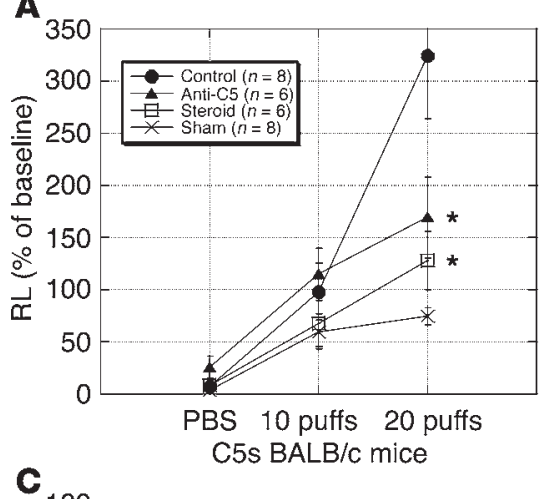

C

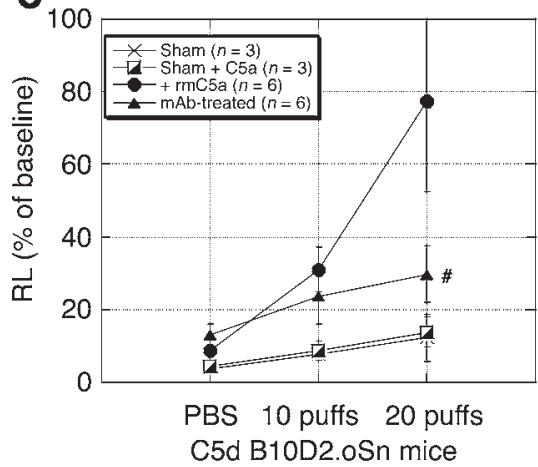

C5d B10D2.oSn mice
B

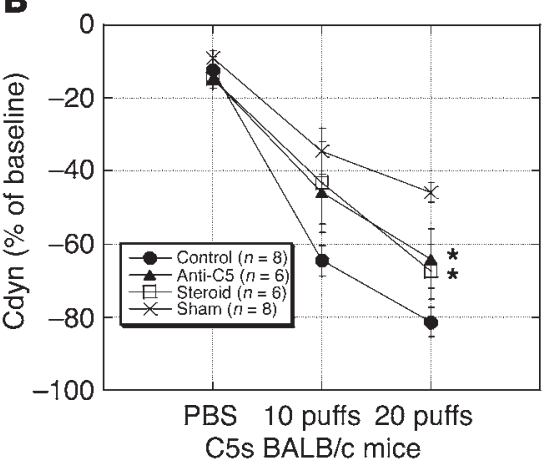

D

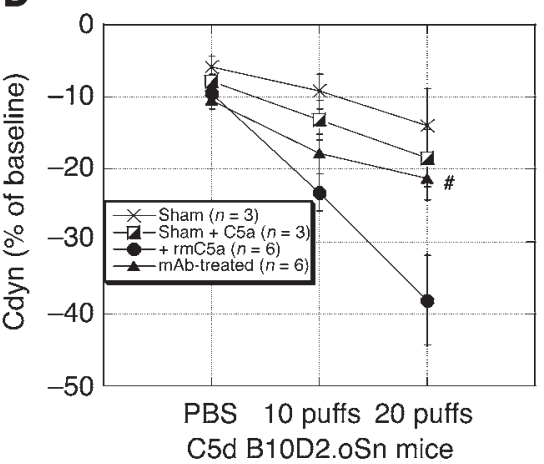

Figure 4

Contribution of C5 at checkpoint 2, the development of AHR. C5s BALB/c mice (A and B) and C5d B10D2.oSn mice (C and D) were immunized with OVA in as demonstrated in checkpoint 2 experiments in Figure 2B. Animals were randomized and given the indicated treatment i.p. on day 33. On day 35, aerosol Mch challenges were administered through a trachea cannula during measurement of $R L(A$ and $\mathbf{C}$ ) and Cdyn (B and $\mathbf{D})$ ). Changes in $R L$ and Cdyn are expressed as a percentage of baseline after each aerosol challenge. Subgroups of C5d mice with or without OVA immunization were reconstituted i.v. with $200 \mu \mathrm{g}$ of rmC5a 3 hours prior to Mch challenges. For C5s BALB/c mice ( $A$ and $\mathbf{B}),{ }^{*} P<0.05$, steroidor anti-C5 mAb-treated compared with control mAb-treated mice; for C5d B10D2.oSn mice (C and D), ${ }^{\#} P<0.05$, mice treated with $\mathrm{mAb}(n=6$ : control mAb, $n=3$; anti-C5 mAb, $n=3)$ compared with OVA-immunized mice reconstituted with $\mathrm{rmC5a}$. There were no statistical differences between $\mathrm{C} 5 \mathrm{~d}$ animals treated with control $\mathrm{mAb}$ and anti-C5 mAb.

doses of aerosol Mch challenges did not induce significant changes in lower airway functions in OVA-immunized B10D2. oSn mice (Figure 4, C and D). Treatment with either anti-C5 $\mathrm{mAb}$ or control $\mathrm{mAb}$ on day 33 had no impact on the development of AHR. The data from both mAb-treated cohorts were pooled as a single mAb-treated cohort (Figure 4, C and D). Furthermore, reconstitution of OVA-immunized B10D2.oSn mice with recombinant mouse $\mathrm{C} 5 \mathrm{a}$ ( $\mathrm{rmC5}$ ) completely restored $\mathrm{AHR}$ in response to aerosol Mch challenge, as indicated by the significant increase in RL and reduction in Cdyn (Figure 4, C and D). Reconstitution with rmC5a was not sufficient for the development of AHR, since sham-treated B10D2.oSn mice reconstituted with rmC5a did not have significant changes in lower airway functions during Mch challenge (Figure 4, C and D). Analysis of serum samples harvested from each mouse after Mch challenges confirmed the C5d status of B10D2.oSn mice, with average hemolytic activity of $8.1 \% \pm 0.9 \%$ compared with $108 \% \pm 3.8 \%$ in $\mathrm{C} 5 \mathrm{~s}$ BALB/c mice. Furthermore, we analyzed random tail samples $(n=2)$ from each group of C 5 s and C5d animals to confirm the $\mathrm{C} 5$ genotype status (data not shown).
Checkpoint 3: contribution of C5 during an ongoing airway response. We next examined the role of C5 in sustaining an ongoing airway response. Animals received intervention during the peak of EAR, which was monitored by DCP at 15 minutes after aerosol exposure to $5 \%$ OVA on day 35 (data not shown). All control mAb-treated BALB/c mice developed severe LAR, with significant increases in RL and reductions in Cdyn 5 hours after allergen provocation (Figure 5, A and B).

We chose to administer treatment through i.v. injection during EAR to achieve rapid systemic C5 inhibition, which completely blocked the development of LAR with minimal increase of RL 5 hours after 5\% OVA provocation (Figure 5A) and prevented much of the loss of lung elasticity, as indicated by the minimal reduction in Cdyn (Figure 5B). Interestingly, corticosteroid treatment did not eliminate the development of LAR, resulting in significant elevation of RL and reduction in Cdyn at 5 hours. Although no immediate impacts (within 5 hours) on lower airway functions were observed after i.v. corticosteroid treatment (Figure 5), this treatment significantly reduced the level of the intrapulmonary IL-13 and significantly improved lower airway functions 24 hours later (data not shown).

Double-blind histologic analysis of lung tissue showed comparable levels of perivascular and peribronchial infiltration of inflammatory cells in the animals 5 hours after i.v. intervention with either corticosteroid, anti-C5 mAb, or control $\mathrm{mAb}$, similar to what is shown in the histological sample in Figure 3F (data not shown).

Effect of C5 inbibition on the migration of inflammatory cells. We next examined the migration of inflammatory cells from airway tissue inflammation into bronchial lumen as enumerated by bronchoalveolar lavage fluid (BALF) wbc analysis. There was a significant reduction in total BALF inflammatory cells in anti-C5 mAb-treated BALB/c mice compared with control mAb-treated mice at checkpoint 1 (Figure 6A). Interestingly, the blockade of migration of inflammatory cells by C5 inhibition exceeded the degree of improved lower airway function (Figure 3, $\mathrm{B}$ and $\mathrm{C}$ ) and reduced tissue inflammation (Figure 3D).

Similarly, when BALF was harvested at checkpoint 3 (Figure 6B), C5 inhibition again significantly blocked the migration of inflammatory cells into bronchial lumen, with significantly lower BALF wbc counts in anti-C5-treated animals than in animals treated with either corticosteroid or control $\mathrm{mAb}$. This result is in dramatic contrast to the significant presence of perivascular and peribronchial infiltration of inflammatory cells in lung tissue harvested before allergen provocation on day 35 (data not shown). The significant blockade of migration of inflammatory cells by C5 inhibition correlated with the significantly enhanced efficacy of C5 inhibition compared with corticosteroid therapy at checkpoint 3 (Figure 5, A and B).

Sham-treated mice provided the baseline level of BALF wbcs, which are primarily normal alveolar macrophages $(82.3 \% \pm 10.3 \%)$ 
A

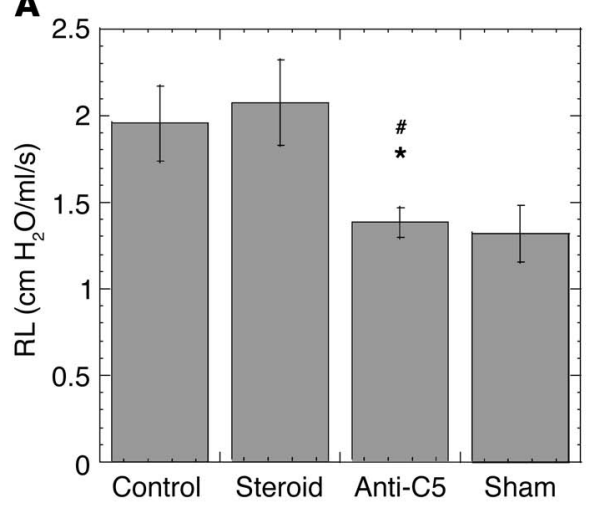

B

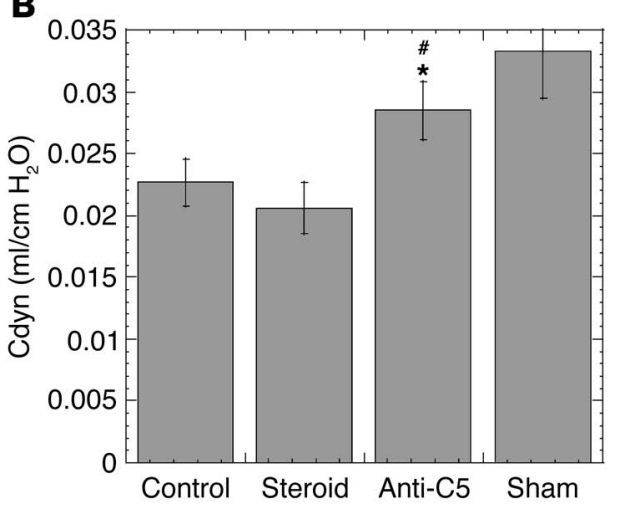

\section{Figure 5}

Contribution of $\mathrm{C} 5$ at checkpoint 3, during an ongoing airway response. Animals that experienced previous airway response to allergen provocation on day 32 were given a second aerosol allergen challenge on day 35. During the peak of EAR, animals were randomized and given the indicated treatment i.v. Animals were trachea cannulated for the measurement of $\mathrm{RL}(\mathbf{A})$ and Cdyn (B) 5 hours after the OVA provocation. $n=7-8$ mice per group. ${ }^{*} P<0.05$ for steroid- or anti-C5 mAb-treated compared with control mAb-treated mice. ${ }^{\#}<0.05$ for corticosteroid-treated compared with anti-C5 mAb-treated mice. according to wbc differential analysis (Figure 6C). Eosinophils constituted more than $45 \%$ of BALF inflammatory cells from all OVAimmunized animals, regardless of the therapeutic intervention. There was a significant reduction in the percentage of neutrophils recovered in BALF from the animals treated at checkpoint 3 with either corticosteroid $(4.5 \% \pm 2.8 \%)$ or anti-C5 $\mathrm{mAb}(6.3 \% \pm 3.3 \%)$ compared with control mAb-treated mice $(18.3 \% \pm 4.4 \%)$. There were no significant reductions in the percentages of eosinophils or lymphocytes in BALF samples from the animals receiving intervention at checkpoint 3 (Figure 6C).

Effect of C5 inhibition on intrapulmonary Th1/Th2 cytokine profile and inflammatory mediators. BALF harvested from control $\mathrm{mAb}$-treated mice had markedly increased levels of IL-5 and IL-13 (Figure 7, $\mathrm{A}$ and $\mathrm{B}$ ) compared with that from sham-treated mice. Corticosteroid treatment markedly reduced the BALF levels of IL-5 and IL-13; however, this did not translate into an immediate improvement in lower airway function (Figure 5, A and B) during the 5 -hour treatment period. The dramatic improvement in lower airway functions (Figure 5) seen after C5 inhibition at checkpoint 3 did not correlate with any reduction of BALF level of IL-5 and IL-13. The statistical difference in BALF levels of IL-13 between anti-C5- and corticosteroid-treated mice was $P=0.051$.

C5 inhibition or corticosteroid therapy at checkpoint 3 did not influence the BALF level of histamine (Figure 7C). This was expected, since histamine had probably already been released into the airway lumen upon the engagement of IgE receptors of mast cells by aerosol allergen provocation and was responsible for the appearance of EAR during therapeutic intervention.

Eosinophils, the most prominent inflammatory cells along lower airways (Figure 3, F-H), are probably responsible for the production of eotaxin, RANTES, and TGF- $\beta$ (Figure $7, \mathrm{D}-\mathrm{F}$ ) in asthmatic individuals. Control $\mathrm{mAb}$-treated animals had significantly elevated BALF levels of eotaxin (Figure 7D), RANTES (Figure 7E), and activated TGF- $\beta$ (Figure 7F), while the levels of these mediators in sham-treated mice were negligible or nondetectable. In contrast, C5 inhibition had a marked impact on BALF levels of eotaxin, RANTES, and activated TGF- $\beta$ when given either at checkpoint 1 (Figure 7, D and E) or checkpoint 3 (Figure 7F). Interestingly, corticosteroid treatment only reduced the BALF level of TGF- $\beta$ when given at checkpoint 3 but had no obvious impact on the production and release of eotaxin or RANTES when given at checkpoint 1 .

Neutrophils are commonly believed to be responsible for the production of bronchial TNF- $\alpha$ and proMMP9. C5 inhibition significantly reduced BALF levels of TNF- $\alpha$ (Figure $7 G$ ) when given at checkpoint 1 , which is significantly different from the results in control mAb-treated mice. The bronchial level of proMMP9 was dramatically reduced and was significantly different from that of control mAb-treated and corticosteroid-treated mice when C5 inhibition was given at checkpoint 3 (Figure $7 \mathrm{H}$ ).
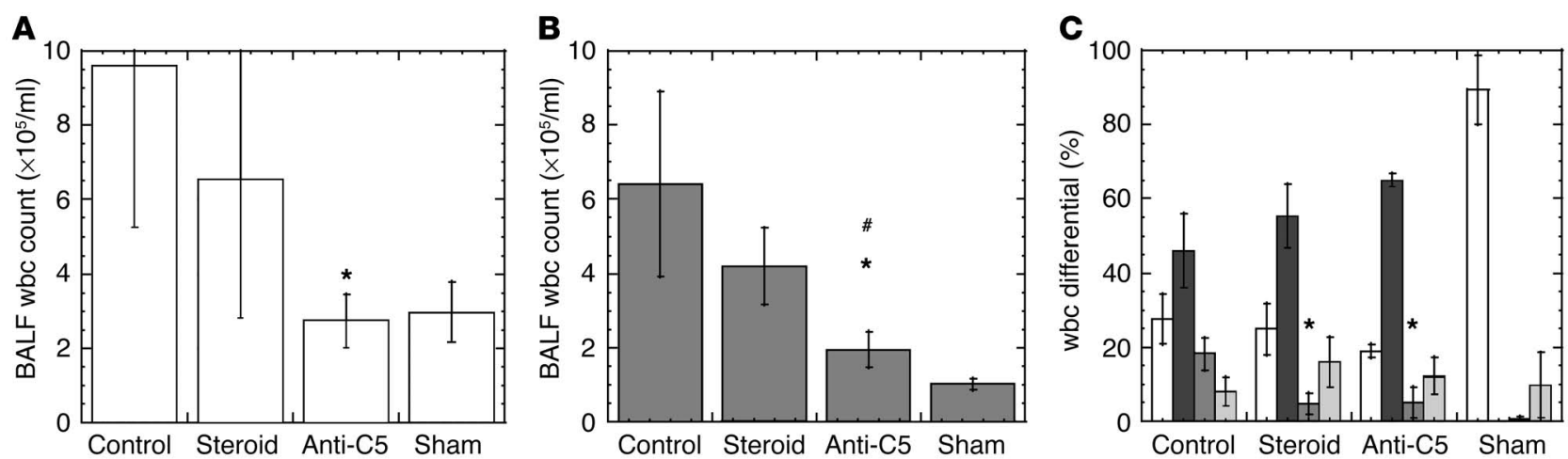

Figure 6

BALF wbc counts and differential analysis. BALF was obtained 5 hours after aerosol allergen provocation at checkpoint 1 (A) or at checkpoint 3 (B and C). Total BALF wbc counts (A and B) and their differential analyses (C) were performed by a pathologist in a double-blind fashion. In $\mathbf{C}$, white bars: alveolar macrophages; dark gray bars: eosinophils; medium gray bars, neutrophils; light gray bars, lymphocytes. $n=6-8 \mathrm{mice}$ per group. ${ }^{\star} P<0.05$ for steroid- or anti-C5 mAb-treated versus control mAb-treated mice. ${ }^{P} P<0.05$ for corticosteroid-treated compared with anti-C5 mAb-treated mice. 

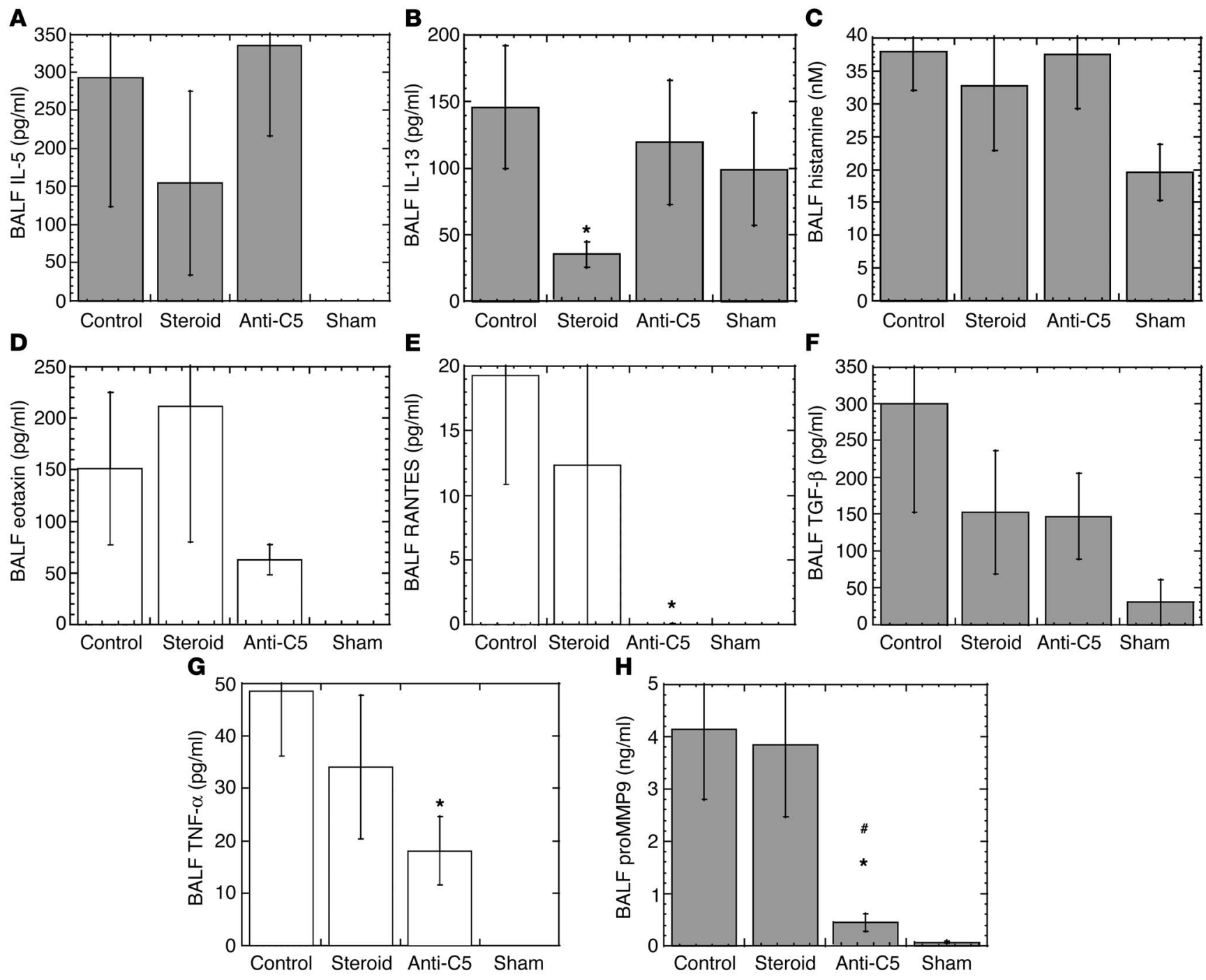

Figure 7

BALF levels of inflammatory mediators. BALF was obtained 5 hours after aerosol allergen provocation at checkpoint 1 (white bars) or checkpoint 3 (gray bars). BALF levels of IL-5 (A), IL-13 (B), histamine (C), eotaxin (D), RANTES (E), activated TGF- $\beta$ (F), TNF- $\alpha$ (G), and proMMP9 (H) were measured by ELISA. $n=6-8$ samples per group. ${ }^{\star} P<0.05$ for steroid- or anti-C5 mAb-treated compared with control mAb-treated mice. $\# P<0.05$ for corticosteroid-treated compared with anti-C5 mAb-treated mice.

Checkpoint 3: contribution of intrapulmonary activation of C5 during an ongoing airway response. The massive migration of inflammatory cells from airway tissue inflammation into bronchial lumen probably results from powerful chemotactic forces deriving from the epithelial mucosa of the airway. We therefore hypothesized that $\mathrm{C} 5$ is activated intrapulmonarily during an airway response to allergen provocation. To test this hypothesis, we evaluated the functions of lower airways and parameters of inflammation after blocking the intrapulmonary activation of C5. We also evaluated the potential impact at checkpoint 3 of inhibitory receptors, such as FcyRIIB $(17,18)$, as the result of interaction between intrapulmonary $\mathrm{C} 5$ and anti-C5 $\mathrm{mAb}$ and the subsequent formation of immune complexes (ICs). We directly compared the effects of intrapulmonary $\mathrm{C} 5$ inhibition with anti-C5 $\mathrm{mAb}$ and anti-C5 Fab in order to achieve this goal. Furthermore, we evaluated the efficacy of an anti-C5a receptor (anti-C5aR) serum $\operatorname{IgG}(\operatorname{sgG})$ in order to dissect the role of intrapulmonary C5a versus C5b-9 at this checkpoint.

As shown in Figure 8, A and B, control mAb-treated animals developed severe LAR with significant increases of RL and significant reductions in Cdyn, in significant contrast to the normal RL and Cdyn seen in sham-treated mice. Aerosol administration of corticosteroid blocked the development of LAR as evidenced by the reduction of RL with corresponding preservation of Cdyn. Blocking intrapulmonary C5 activation with aerosol administration of either anti-C5 mAb or anti-C5 Fab during the peak of EAR also prevented the development of LAR, with minimal increase in $\mathrm{RL}$ and minimal reduction in Cdyn (Figure 8, A and B). RL and Cdyn of both anti-C5-treated cohorts were significantly different from those of control mAb-treated animals. Blocking the binding of intrapulmonary C5a to its receptors with an anti-C5aR sIgG also prevented the development of LAR, with minimal increase 
A
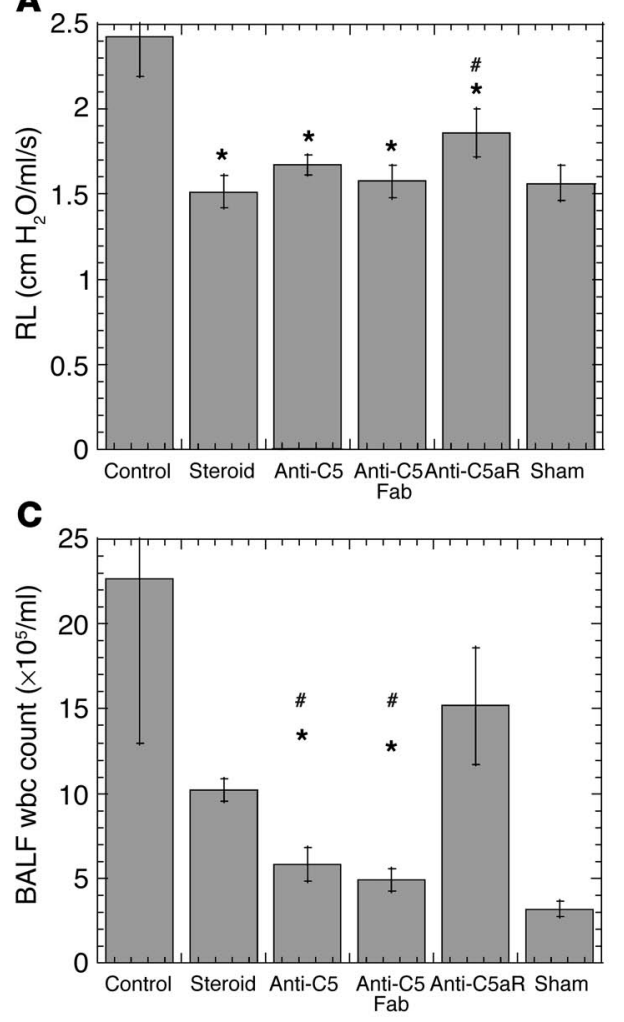

B

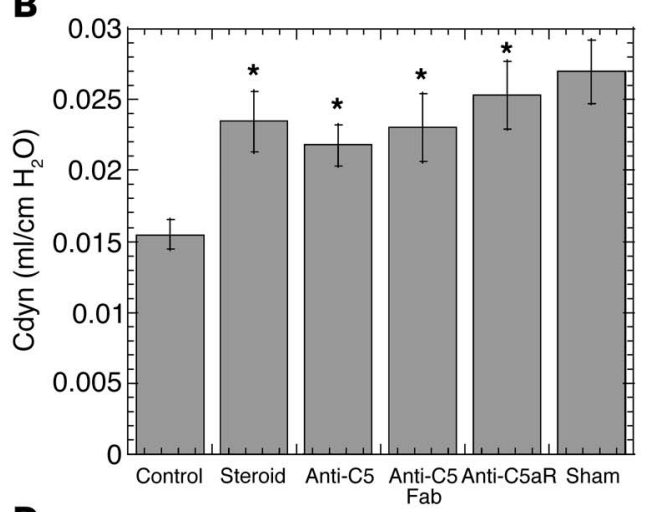

D

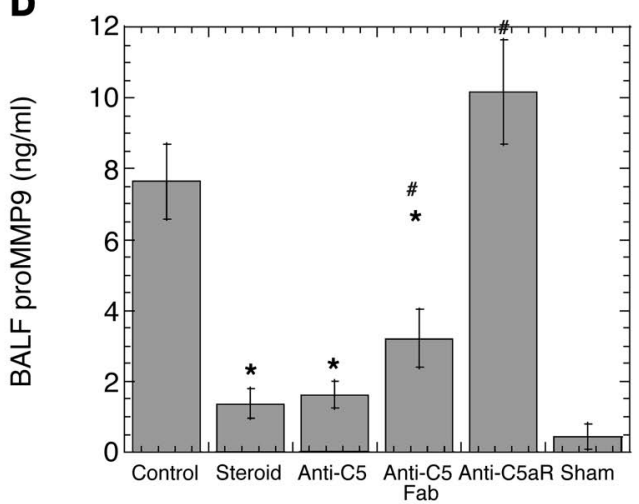

\section{Figure 8}

Contribution of intrapulmonary C5 activation at checkpoint 3 . Results represent data pooled from 3 separate experiments with identical experimental procedures. Animals were aerosol challenged with $5 \%$ OVA and randomized. During EAR, animals were then given aerosol administration of 1 of the indicated treatments through a jet nebulizer. Sham-immunized mice were treated with aerosol PBS. All animals were trachea cannulated for the measurement of $R L(A)$ and Cdyn (B) 5 hours after the OVA provocation. BALF wbc counts (C) and proMMP9 levels (D) were analyzed in the majority of animals. The data from control mAb- $(n=15)$ and control slgG-treated mice $(n=4)$ were pooled together. anti-C5 Fab: $n=3$; animals in all other cohorts: $n=6-19 .{ }^{*} P<0.05$ for steroid-, antiC5 mAb-, anti-C5 Fab-, or antiC5aR slgG-treated compared with control mAb-treated mice. ${ }^{\#} P<0.05$ for corticosteroid-treated compared with anti-C5 mAb-, anti-C5 Fab-, or anti-C5aR slgG-treated mice. in $\mathrm{RL}$ and minimal reduction in Cdyn, which is similar what was observed in animals treated with anti-C5 $\mathrm{mAb}$. There was no statistical difference between the cohorts treated with anti-C5 $\mathrm{mAb}$, anti-C5 Fab, or anti-C5aR sIgG (Figure 8, A and B).

Interestingly, when the parameters of intrapulmonary inflammatory activities were examined, such as the migration of inflammatory cells (Figure 8C) and the BALF level of proMMP9 (Figure 8D), significant differences between these activities were observed depending on whether $\mathrm{C} 5$ activation or the binding of C5a to C5aR was blocked. Aerosol administration of either anti-C5 mAb or its Fab fragment during EAR significantly blocked both the migration of inflammatory cells into bronchial airway lumen and the elevation of the BALF level of proMMP9, whereas blocking the engagement of C5aR had no significant impact on these 2 key inflammatory parameters, which is similar to what was observed in the animals treated with control $\mathrm{mAb}$ (Figure 8, C and D). Consistent with its ability to block the development of LAR (Figure 8, A and B), aerosol administration of corticosteroid also ameliorated intrapulmonary inflammatory activities, resulting in marked reduction in the migration of inflammatory cells and the production of proMMP9 (Figure 8, C and D), which indicates the importance of inflammatory activities at the epithelial side of the bronchial lumen.

Aerosol administration of anti-C5 mAb, anti-C5 Fab, or antiC5aR sIgG did not have any impact on serum C5b-9-mediated hemolytic activity (data not shown).

\section{Discussion}

We examined the contribution of $\mathrm{C} 5$ and its activated components at 3 essential checkpoints during the course of asthma. First, we showed that $\mathrm{C} 5$ contributes to the initiation of airway inflammation. We next demonstrated the critical contribution of C5 in the development of AHR in animals with established airway inflammation. In dramatic contrast to a previous report (7), we demonstrated that C5d B10D2.oSn mice did not develop AHR to aerosol Mch challenges. Reconstitution of $\mathrm{rmC5}$ a restored AHR in OVA-immunized C5d mice. Finally, we demonstrated the significant role of activated $\mathrm{C} 5$ components in sustaining an ongoing airway response to allergen challenge. We hypothesized that intrapulmonarily activated C5 components arise primarily from the cleavage of C5 into C5a and C5b-9 at the epithelial side of the airway mucosa after exposure to allergen or infections, which results in massive migration of inflammatory cells into airway lumen and triggers the production as well as the release of harmful inflammatory mediators.

One of the hallmarks of asthma is the development of airway inflammation. Individuals with a genetic predisposition may have modulated adaptive immune responses to environmental exposures, including activation of allergen-specific $\mathrm{CD} 4^{+} \mathrm{T}$ cells and polarization of Th2 cytokines, such as IL-4 and IL-5, which are critically involved in the production of allergen-specific IgE and the recruitment of eosinophils (14). More recently, IL-13 was identified as a key player in the pathogenesis and reported to be both necessary and sufficient to induce the airway response in animals (19). As expected, the broad spectrum of antiinflammatory activities of corticosteroid, including modulating the Th2 cytokine profile (Figure 7, A and B) and blocking the recruitment of inflammatory cells into lower airways (Figure 3D), correlates with its potent antiasthmatic activity. Consistent with its antiinflammatory activities reported earlier (20), C5 inhibition at checkpoint 1 resulted in significantly less airway inflammation without significant impact on the adaptive immune system's responses to allergen exposures. Interestingly, C5d and C5s animals developed airway inflammation to a similar degree. One possible explanation is that airways 
are directly exposed to allergens and infections. These exposures may activate other complement components, such as C3a, which has very similar proinflammatory properties $(5,6)$ and was known to contribute to the pathogenesis (8). These data indicated that activated C5 components are critically involved in but are not necessary for the initiation of airway inflammation, which requires the activation of adaptive immune responses to aerosol exposures of allergen (14). However, at checkpoints 2 and 3, when airway inflammation was already well established, our data indicated that C5 inhibition had similar or better efficacy than corticosteroid treatment in ameliorating airway inflammation and improving lower airway functions (Figures 4-8).

The second hallmark of asthma, the development of AHR in response to nonspecific stimuli, was selected as another critical checkpoint. By prescreening several strains of C5s and C5d mice, we eliminated the genetic influences on the function of muscarinic acetylcholine receptor responsible for intrinsic AHR $(15,16)$ in this study. With the use of animals that do not have intrinsic AHR, C5 inhibition was sufficient to prevent the development of AHR to aerosol Mch challenges in C5s BALB/c mice with established airway inflammation (Figure 4, A and B), while reconstitution of $\mathrm{C} 5 \mathrm{~d} \mathrm{~B} 10 \mathrm{D} 2 . \mathrm{oSn}$ mice with $\mathrm{rmC} 5$ a restored AHR, also in the presence of established airway inflammation (Figure 4, C and D). These data suggested that activated C5 components, most likely $\mathrm{C} 5 \mathrm{a}$, serve as a direct link between the innate immune responses and the key components of the airways responsible for AHR. C5aR was found on airway smooth muscle cells and airway epithelia (21). Although the direct interaction of activated C5 components, in particular C5a, with receptors on airway smooth muscles and epithelium was demonstrated previously $(13,22)$, our data indicated that the maintenance of AHR depends upon complex interactions among airway epithelia, airway smooth muscle cells, inflammatory cells, and their inflammatory mediators, as suggested by minimal impact on the functions of lower airways during Mch challenges after systemic reconstitution (i.v.) of sham-treated B10D2.oSn mice with rmC5a (Figure 4, C and D). In contrast, results of a study using C5d strains that were known to have intrinsic AHR to various nonspecific stimuli without allergen sensitization and the presence of airway inflammation $(15,16)$ led Karp et al. to the opposite conclusion regarding the role of C5 in asthma (7).

The ongoing airway responses after aerosol allergen provocation was selected as the third critical checkpoint. The development of EAR and LAR together with massive production and release of multiple inflammatory mediators provides a good opportunity to examine the complex interaction of activated C5 components with other components of innate and adaptive immune responses and their role in pathogenesis.

Although we have previously demonstrated that in vivo administration of anti-C5 $\mathrm{mAb}$ has no impact on the function of $\mathrm{Fc}$ receptors (20), the potential impact at checkpoint 3 by inhibitory receptors, such as Fc $\gamma$ RIB $(17,18)$, as the result of interaction between intrapulmonary $\mathrm{C} 5$ and anti-C $5 \mathrm{mAb}$ and the subsequent formation of ICs was further evaluated. C5 inhibition by either anti-C5 mAb or its Fab fragment achieved similar in vivo efficacy in ameliorating intrapulmonary inflammatory activities as well as in improving functions of lower airways. These data clearly demonstrated that C5 inhibition, other than activation of inhibitory receptors by the formation of intrapulmonary ICs, accomplished the above biological activities.
Our data also suggest that in the presence of airway inflammation, activated C5 components function as the key regulators of the downstream inflammatory cascade. This regulatory effect was demonstrated by C5's ability to influence the migration of inflammatory cells into airway lumen as well as the activation and the release of multiple harmful mediators corresponding to the significant changes of lower airway functions at checkpoint 3. C5a is probably the most potent activator of inflammatory cells (5), and C5aR has been identified on circulating leukocytes, mast cells, macrophages, and endothelial cells (23). The disassociation of significant improvement of lower airway functions from significant presence of intrapulmonary inflammatory activities after antiC5aR sIgG treatment is consistent with the hypothesis that the direct engagement of $\mathrm{C} 5 \mathrm{a}$ with its receptors expressed on airway smooth muscle cells and epithelia is, in part, responsible for airway constriction (22). Our data also indicate that the chemotactic activity and cell activation properties of C5b-9 (24) are responsible for the significant intrapulmonary inflammatory activities in the absence of $\mathrm{C} 5 \mathrm{aR}$ engagement. In the presence of established airway inflammation and ICs, C5b-9 can sufficiently regulate the downstream inflammatory cascade by triggering releases of multiple mediators such as proMMP9 independently of the engagement of C5a with its receptors expressed on inflammatory cells (Figure 8D). The presence of elevated levels of C5b-9 together with the presence of multiple inflammatory mediators contributed to migration of inflammatory cells into the bronchial lumen in animals treated with anti-C5aR sIgG (Figure 8C).

Both the adaptive and innate immune systems contributed in the complex process of the production and release of multiple inflammatory mediators, including leukotrienes, prostaglandins, histamine, alkaline proteins, cytokines, chemokines, and enzymes, which are responsible for bronchospasm, altered vascular permeability, adherence and migration of inflammatory cells, mucosal edema, and excessive mucous secretion and ultimately led to 3 unique but interdependent phenomena: airway inflammation, airway obstruction, and AHR. Since there are many redundant biological mechanisms, under the right circumstances, such as in genetically deficient animals, these redundant mechanisms may compensate and contribute in a more significant fashion. The complex relationship and interactions of these components responsible for airway inflammation, obstruction, and AHR, such as the correspondence between the level of intrapulmonarily activated complement components and subsequent release of harmful mediators, deserve further investigation.

Lung parenchymal cells possess the ability to produce $\mathrm{C} 5$ and cleave $\mathrm{C} 5$ into activated fragments (25). The activation of $\mathrm{C} 5$ as part of innate immune responses may well be an innocuous by-product of a self-defensive response, due in part to direct enzymatic cleavage of C5 (26) after exposure to allergens (6), house dust (26), smoke (27), or airway infections (1-3). The formation of intrapulmonary ICs after allergen exposure may also activate complement cascade (13). According to this hypothesis, activated C5 components are primarily located at the epithelial side of the airway, and, in nonasthmatic individuals, the intrapulmonary activation of complement is well regulated (28). Consistent with this hypothesis, our data demonstrated that intrapulmonary $\mathrm{C} 5$ inhibition achieved an efficacy similar to that of systemic C5 inhibition at checkpoint 3 (Figure 8) and at checkpoint 2 (unpublished observations). These data further suggest that intrapulmonarily activated C5 components are major proinflammatory rather than antiinflammatory forces. 
We have demonstrated the striking effect of C5 inhibition on the inflammatory mediators produced or released by neutrophils or eosinophils (Figure 7). These inflammatory mediators, in particular TGF- $\beta$, RANTES, and proMMP9, may cause irreversible damage to airways by inducing bronchial smooth muscle hypertrophy and deposition of collagen under the basal membranes of respiratory epithelium, which leads to the remodeling and repair of lower airways. This repair and remodeling of lower airways may result in poor long-term prognosis in patients with severe asthma (29). Currently, the impact of corticosteroid on the production of these harmful mediators and on airway remodeling remains controversial (29). Therefore, it is arguable that C5 inhibition, which utilizes a unique and different antiinflammatory mechanism, may complement the potent antiinflammatory effect of corticosteroid and have an added benefit for long-term prognosis.

This study clearly demonstrates that the complex functional changes in airways seen in individuals with asthma coexist with the complex inflammatory processes. On one hand, activated C5 components, such as $\mathrm{C} 5$ a through its binding with airway $\mathrm{C} 5 \mathrm{aR}$, serve as the direct link between the innate immune system and AHR. On the other hand, both C5a and C5b-9 regulate the downstream inflammatory cascade through their chemotactic and cell activation activities in the presence of ICs and established airway inflammation. Blocking the generation of $\mathrm{C} 5 \mathrm{a}$ and $\mathrm{C} 5 \mathrm{~b}-9$ not only improves the functioning of lower airways but also ameliorates intrapulmonary inflammatory activities. In contrast, C5aR antagonist significantly improves functions of lower airways without having a dramatic impact on intrapulmonary inflammatory activities. This study also suggests that the potential synergistic effect of $\mathrm{C} 5$ inhibition and corticosteroid should be further investigated and that targeting the innate immune system, as by blocking the intrapulmonary activation of $\mathrm{C} 5$, is a potential clinical approach for treating patients with asthma.

\section{Methods}

Animals. Male C5s BALB/cByJ and C57BL/6J mice and C5d B10D2oSn/J, $A K R / J$, and SWR/J mice were purchased from the Jackson Laboratory and housed in a pathogen-free facility. In addition to the analysis of serum C5b-9-mediated hemolysis, the C5 genotype was further confirmed by PCR performed on tail DNA using a pair of primers (5'-CACGATAATGGGAGTCATCTGGG-3' and 5'-AAGTTGGAGTGTGGTCTTTGGGCC-3') that amplify a 280-bp DNA fragment from both C5s and C5d DNA. This fragment encodes a HindIII site that is selectively destroyed by the mutation in the C5 gene, such that HindIII (New England BioLabs) digestion selectively cleaves the C5s but not the C5d PCR products into 150- and 130-bp fragments. All animal protocols were reviewed by the Alexion Institution Animal Care and Use Committee and were in accordance with NIH guidelines.

Reagents. Anti-mouse C5 mAb (BB5.1) (20) and an isotype-matched irrelevant control $\mathrm{mAb}$ (HFN7.1), which is specific to human fibronectin, were purified from ascites. BB5.1 Fab, which was purified with a kit (ImmunoPure IgG1 Fab Preparation kit; Pierce), had retained more than $90 \%$ of activity of BB5.1 as indicated by in vitro hemolytic assay and was more than $97 \%$ in purity. The anti-C5aR sIgG, which was shown to prevent mortality in experimental sepsis (30), was able to prevent zymosan-induced neutrophil migration. Both anti-C5aR sIgG and the control sIgG were purified from sera harvested from C5aR-deficient mice (8) that were repeatedly immunized with either PBS or peptide spanning the $\mathrm{N}$ terminus of the mouse C5aR and 1 extra cysteine (30) emulsified with CFA. Antibodies from either ascites or serum samples were purified by protein A affinity chromatography (Amersham Biosciences). Corticosteroid (dexamethasone) was purchased from Sigma-Aldrich. rmC5a, which was free of endotoxin contamination and had potent chemotactic activity in neutrophil migration assay, was cloned and purified as previously reported (30).

Induction of airway inflammation and severe airway response. BALB/c and B10D2.oSn mice 10-12 weeks of age were sensitized on days 1 and 14 with i.p. injection of $20 \mu \mathrm{g}$ OVA (Grade V; Sigma-Aldrich) emulsified in $2 \mathrm{mg}$ aluminum hydroxide (Imject Alum; Pierce). Mice were immunized with $1 \%$ OVA for 10 minutes via airway on days 28,29 , and 30 with a jet nebulizer (Harvard Apparatus Inc.) connected to a single housing chamber (Buxco Electronics Inc.). On day 32, all sensitized animals were aerosol challenged with $5 \%$ OVA for 10 minutes. In checkpoint 3 experiments, $\mathrm{BALB} / \mathrm{c}$ mice were again aerosol challenged with $5 \%$ OVA on day 35 . Sham C 5 s or C5d mice were immunized with alum only and aerosol challenged with PBS instead of OVA.

Noninvasive measurement of sRaw. sRaw, a measure of the resistance of both upper and lower airways, was determined by aCP in conscious animals with spontaneous breathing (Buxco Electronics Inc.). The DCP was used to monitor the longitudinal changes in sRaw prior to and after each 5\% OVA aerosol challenge. In checkpoint 3 experiments, a DCP was used to ensure the appearance of EAR prior to randomizing animals into different treatment groups.

Invasive measurement of lower airway functions. Buxco BioSystem XA for Windows software and a Buxco whole body plethysmograph were used to measure changes in RL and Cdyn. Mice were anesthetized (2,2,2-tribromoethanol [Avertin], $160 \mathrm{mg} / \mathrm{kg}$; Sigma Aldrich) and tracheas cannulated. Spontaneous breathing was blocked by pancuronium bromide $(0.3 \mathrm{mg} / \mathrm{kg}$, i.p.). Respirations were maintained by a Harvard Apparatus Inspira ventilator, which calculated a tidal volume and respiratory rate according to body weight. Measurements of RL and Cdyn were performed during the peak of LAR, 5 hours after aerosol challenge with 5\% OVA. Real-time changes in RL and Cdyn were recorded by Buxco Biosystem SA for Windows software and reported as the mean value of 5 minutes of recording.

AHR was evaluated based on changes in RL and Cdyn during aerosol Mch challenges. Changes in RL and Cdyn were expressed as a percentage of baseline after each aerosol challenge. A Buxco aerosol control and sonicating nebulizer unit was attached to the ventilator for aerosol delivery of Mch through tracheal cannulation. PBS or Mch $(1.6 \mathrm{mg} / \mathrm{ml})$ was delivered at the rate of 10 or 20 puffs per 10 seconds, with each puff of aerosol delivery lasting $15 \mathrm{~ms}$.

C5 inhibition. Checkpoint 1: For C5 inhibition at checkpoint 1, anti-C5 $\mathrm{mAb}$ (BB5.1) or a control mAb (HFN7.1) was administered i.p. at $40 \mathrm{mg} / \mathrm{kg}$ on days 25,29 , and 31 . Dexamethasone $(2 \mathrm{mg} / \mathrm{kg})$ was used as a positive control. Checkpoint 2: Animals that experienced airway response to $5 \%$ OVA provocation on day 32 were randomized for the following studies. For C5 inhibition at checkpoint 2, animals were given 1 i.p. injection of either dexamethasone ( $2 \mathrm{mg} / \mathrm{kg})$, anti-C5 $\mathrm{mAb}(40 \mathrm{mg} / \mathrm{ml})$, or control $\mathrm{mAb}(40 \mathrm{mg} / \mathrm{kg})$ on day 33 . On day 35 , animals were anesthetized and tracheas cannulated for measurement of RL and Cdyn during aerosol Mch challenges. Checkpoint 3: At checkpoint 3, anti-C5 mAb (40 mg/kg), control $\mathrm{mAb}(40 \mathrm{mg} / \mathrm{kg})$, or dexamethasone $(2 \mathrm{mg} / \mathrm{kg})$ was administered by i.v. injection 20 minutes after animals received aerosol challenge with 5\% OVA on day 35 . For intrapulmonary $\mathrm{C} 5$ inhibition at this critical point, animals were given aerosol administration of either anti-C $5 \mathrm{mAb}(3 \mathrm{mg} / \mathrm{ml})$, anti-C5 Fab (3 mg/ml), control mAb (3 mg/ml), anti-C5aR sIgG (3 mg/ml), control sIgG $(2 \mathrm{mg} / \mathrm{ml})$, or corticosteroid $(2 \mathrm{mg} / \mathrm{ml})$ for $10-30$ minutes using a jet nebulizer connected to a single chamber housing the animal. Sham-immunized mice were given treatment with the same volume of PBS solution administered through the same route. The results from 3 intrapulmonary C5 inhibition experiments, which were conducted with identical protocols, were pooled. 
Analysis of BALF. Approximately 5.5 hours after allergen provocation, after the measurement of RL and Cdyn, we harvested BALF by instilling $1 \mathrm{ml}$ PBS through the tracheal cannula, followed by cannula and gentle aspiration. The BALF was resuspended in $400 \mu \mathrm{l}$ PBS. We counted the total numbers of wbc in BALF using a hemocytometer or an automatic cell counter as in the aerosol experiments (CELL-DYN 3700; Abbott Laboratories). Diff-Quik (VWR International) was used to prepare, fix, and stain cytospin slides. The wbc differential was determined by a certified pathologist after counting a total of $300 \mathrm{wbc}$ per slide on a $\times 100$ microscopic lens. The BALF levels of proMMP9, activated TGF- $\beta$, RANTES, eotaxin, and IL-13 (R\&D Systems), IL-5 (Amersham Pharmacia Biotech Inc.), and histamine (Beckman Coulter) were measured by ELISA according to the manufacturers' instructions.

Analysis of serum OVA-specific antibodies. Serum samples were harvested 5 hours after $5 \%$ allergen provocation on day 32 for OVA-specific IgG and IgE by ELISA.

Lung histology. Lung was inflated with $10 \%$ buffered formalin $(1 \mathrm{ml})$ through tracheal cannulation and fixed in $10 \%$ formalin at least 24 hours. Lung samples were stained with Giemsa. Double-blind histological analysis was performed to quantify airway inflammation according to the following criteria: 0 , no detectable airway inflammation; 1 , less than $25 \%$ bronchials and surrounding vasculature were found to have either perivascular or peribronchial inflammatory cell infiltration; 2 , approximately $25-50 \%$ of bronchials and surrounding vasculature were affected; 3 , approximately $50-75 \%$ bronchials and surrounding

1. Nagy, A., et al. 2003. The development of asthma in children infected with Chlamydia pneumoniae is dependent on the modifying effect of mannose-binding lectin. J. Allergy Clin. Immunol. 112:729-734.

2. Kasamatsu, Y., et al. 1993. Experimental acute lung injury in guinea pigs after aerosol challenge with sonicated Pseudomonas aeruginosa whole cells. Arerugi. 42:1616-1622.

3. Bjornson, A.B., Mellencamp, M.A., and Schiff, G.M. 1991. Complement is activated in the upper respiratory tract during influenza virus infection. Am. Rev. Respir. Dis. 143:1062-1066.

4. Lachmann, P.J. 1996. Current topics in complement research. Res. Immunol. 147:69-70.

5. Takafuji, S., Tadokoro, K., Ito, K., and Dahinden, C.A. 1994. Degranulation from human eosinophils stimulated with C3a and C5a. Int. Arch. Allergy Immunol. 104(Suppl. 1):27-29.

6. Nagata, S., and Glovsky, M.M. 1987. Activation of human serum complement with allergens. I. Generation of C3a, C4a, and C5a and induction of human neutrophil aggregation. J. Allergy Clin. Immunol. 80:24-32.

7. Karp, C.L., et al. 2000. Identification of complement factor 5 as a susceptibility locus for experimental allergic asthma. Nat. Immunol. 1:221-226.

8. Gerard, N.P., and Gerard, C. 2002. Complement in allergy and asthma. Curr. Opin. Immunol. 14:705-708.

9. Abe, M., et al. 2001. Contribution of anaphylatoxin C5a to late airway responses after repeated exposure of antigen to allergic rats. J. Immunol. 167:4651-4660.

10. Lukacs, N.W., Glovsky, M.M., and Ward, P.A. 2001. Complement-dependent immune complex-induced bronchial inflammation and hyperreactivity. Am. J. Physiol. Lung Cell Mol. Physiol. vasculature were affected; 4 , more than $75 \%$ of bronchials and surrounding vasculature were affected.

C5b-9-mediated hemolytic assay and in vitro C5a-mediated neutrophil migration assay. Serum harvested at the indicated time was used as the source of C5b-9, which mediated the hemolysis of chicken rbc as previously reported (20). Mouse sera (Sigma-Aldrich) were incubated with either BB5.1 $(100 \mu \mathrm{g} / \mathrm{ml})$ or the same volume of PBS prior to activation with zymosan $(1 \mathrm{mg} / \mathrm{ml}$; Sigma-Aldrich). Diluted sera (20\%) were used as the source of C5a for neutrophil chemotaxis experiments.

Statistical analysis. The data are expressed as mean \pm SEM. Student's 1-tailed $t$ test assuming equal variance was used (Excel; Microsoft Corp.). $P<0.05$ was considered statistically significant.

\section{Acknowledgments}

We thank Stephan Saxe and David Stenger for critical review of the manuscript and Susan Liu Chen, Christine Siever, Jane Kristan, and Yamin Shen for technical support. We also thank Phil Askenase for helpful discussion.

Received for publication August 3, 2004, and accepted in revised form April 12, 2005.

Address correspondence to: Yi Wang, Alexion Pharmaceuticals Inc., 352 Knotter Drive, Cheshire, Connecticut 06410, USA. Phone: (203) 271-8247; Fax: (203) 271-8195; E-mail:wangy@alxn.com.
280:L512-L518.

11. Gonczi, Z., et al. 1997. The severity of clinical symptoms in ragweed-allergic patients is related to the extent of ragweed-induced complement activation in their sera. Allergy. 52:1110-1114.

12. Vakeva, A.P., et al. 1998. Myocardial infarction and apoptosis after myocardial ischemia and reperfusion: Role of the terminal complement components and inhibition by anti-C5 therapy. Circulation. 97:2259-2267.

13. Larsen, G.L. 1985. Hypersensitivity lung disease. Annu. Rev. Immunol. 3:59-85.

14. Cieslewicz, G., et al. 1999. The late, but not early, asthmatic response is dependent on IL-5 and correlates with eosinophil infiltration. J. Clin. Invest. 104:301-308.

15. De Sanctis, G.T., and Drazen, J.M. 1997. Genetics of native airway responsiveness in mice. Am. J. Respir. Crit. Care Med. 156:S82-S88.

16. Levitt, R.C., and Mitzner, W. 1988. Expression of airway hyperreactivity to acetylcholine as a simple autosomal recessive trait in mice. FASEB J. 2:2605-2608.

17. Katz, H.R. 2002. Inhibitory receptors and allergy. Curr. Opin. Immunol. 14:698-704.

18. Ravetch, J.V., and Bolland, S. 2001. IgG Fc receptors. Annu. Rev. Immunol. 19:275-290.

19. Wills-Karp, M., et al. 1998. Interleukin-13: central mediator of allergic asthma. Science. 282:2258-2261.

20. Wang, Y., et al. 1996. Amelioration of lupus-like autoimmune disease in NZB/WF1 mice after treatment with a blocking monoclonal antibody specific for complement component C5. Proc. Natl. Acad. Sci. U. S. A. 93:8563-8568.

21. Drouin, S.M., et al. 2001. Expression of the complement anaphylatoxin C3a and C5a receptors on bronchial epithelial and smooth muscle cells in models of sepsis and asthma. J. Immunol. 166:2025-2032.
22. Irvin, C.G., Berend, N., and Henson, P.M. 1986. Airways hyperreactivity and inflammation produced by aerosolization of human C5A des arg. Am. Rev. Respir. Dis. 134:777-783.

23. Chenoweth, D.E., and Hugli, T.E. 1978. Demonstration of specific C5a receptor on intact human polymorphonuclear leukocytes. Proc. Natl. Acad. Sci. U. S. A. 75:3943-3947.

24. Czermak, B.J., et al. 1999. Synergistic enhancement of chemokine generation and lung injury by C5a or the membrane attack complex of complement. Am. J. Pathol. 154:1513-1524.

25. Desai, U., Dickey, B., Varani, J., and Kreutzer, D.L. 1984. Demonstration of C5 cleaving activity in bronchoalveolar fluids and cells: a mechanism of acute and chronic alveolitis. J. Exp. Pathol. 1:201-216.

26. Maruo, K., Akaike, T., Ono, T., Okamoto, T., and Maeda, H. 1997. Generation of anaphylatoxins through proteolytic processing of C3 and C5 by house dust mite protease. J. Allergy Clin. Immunol. 100:253-260.

27. Robbins, R.A., Nelson, K.J., Gossman, G.L., Koyama, S., and Rennard, S.I. 1991. Complement activation by cigarette smoke. Am. J. Physiol. 260:L254-L259.

28. Varsano, S., Kaminsky, M., Kaiser, M., and Rashkovsky, L. 2000. Generation of complement C3 and expression of cell membrane complement inhibitory proteins by human bronchial epithelium cell line. Thorax. 55:364-369.

29. Boulet, L.P., et al. 2000. Airway hyperresponsiveness, inflammation, and subepithelial collagen deposition in recently diagnosed versus long-standing mild asthma. Influence of inhaled corticosteroids. Am. J. Respir. Crit. Care Med. 162:1308-1313.

30. Riedemann, N.C., et al. 2002. Increased C5a receptor expression in sepsis. J. Clin. Invest. 110:101-108. doi:10.1172/JCI200215409. 